\title{
La lectura no utilitaria en la universidad en la era digital. Un análisis multivariante que ubica el texto impreso en la lectura de literatura
}

\author{
A. Olivia Jarvio Fernández \\ Universidad Veracruzana, México \\ ojarvio@uv.mx \\ Mario Miguel Ojeda Ramírez \\ Universidad Veracruzana, México \\ mojeda@uv.mx
}

Cita sugerida: Fernández , A. O. J. y Ojeda Ramírez, M. M. (2018). La lectura no utilitaria en la universidad en la era digital. Un análisis multivariante que ubica el texto impreso en la lectura de literatura. Palabra Clave (La Plata), 7(2), e051. https://doi.org/10.24215/18539912e051 http://creativecommons.org/licenses/by-nc-sa/4.0/deed.es_AR 


\title{
La lectura no utilitaria en la universidad en la era digital. Un análisis multivariante que ubica el texto impreso en la lectura de literatura
}

Non-utilitarian reading in the university in the digital age. A multivariate analysis that locates the printed text in the literature reading

A. Olivia Jarvio Fernández

Universidad Veracruzana, México

ojarvio@uv.mx

Mario Miguel Ojeda Ramírez

Universidad Veracruzana, México

\section{Resumen:}

En este trabajo se explora la preferencia del texto impreso para la lectura de literatura en una comunidad universitaria. Se hace una caracterización de la lectura y la escritura en la universidad y se presenta una revisión de la importancia de la lectura no utilitaria en este contexto. Se revisa el tema de la apropiación de las TIC en el ámbito universitario, identificando la relevancia de la lectura y la escritura en la era digital. El estudio de caso usa datos de una encuesta aplicada en la Universidad Veracruzana, para lo que se presenta el contexto y los antecedentes. Se hace una descripción de las variables y la estrategia de análisis estadístico. Se analizan los factores que están asociados a la preferencia de formato y se caracterizan las principales asociaciones a partir de pruebas de independencia usando el estadístico Ji-Cuadrado; para las asociaciones multivariantes se corrió un análisis de correspondencia múltiple. Se concluye que la preferencia del texto impreso sigue siendo muy marcada, un poco más entre las mujeres, destacándose el nivel de gusto y el tiempo de dedicación entre los académicos y los funcionarios. No obstante, el concepto de lectura que es más frecuente se relaciona con la lectura utilitaria.

PALABRAS CLAVE: Promoción de la lectura; Encuestas sobre lectura; Lectura utilitaria; Lectura por placer.

\begin{abstract}
:
This paper explores the preference of the printed text for reading literature in a university community. A characterization of reading and writing in the university is made and a review of the importance of non-utilitarian reading is presented in this context. The issue of the appropriation of ICT in the university environment is reviewed, identifying the relevance of reading and writing in the digital age. The case study uses data from a survey applied at the Universidad Veracruzana, for which the context and background are presented. A description of the variables and the strategy of statistical analysis is made. We analyze the factors that are associated with the format preference and characterize the main associations based on independence tests using the Chi-Square statistic; for multivariate associations, a multiple correspondence analysis was run. It is concluded that the preference of the printed text is still very marked, a little more among women, highlighting the level of taste and time of dedication among academics and officials. However, the concept of reading that is more frequent is related to the utilitarian reading.
\end{abstract}

KEYWORDS: Reading promotion, Reading surveys, Utilitarian reading, Reading for pleasure,

\section{INTRODUCCIÓN}

La lectura es una actividad inherente a la universidad. Más generalmente, es posible aseverar que las instituciones de educación superior (IES) -como formadoras de profesionales e impulsoras de la generación, aplicación y difusión del conocimiento, así como de la vinculación y extensión de los servicios a los diferentes sectores de la sociedad- tienen a esta actividad como cotidiana y fundamental: no se cuestiona si los estudiantes, académicos, funcionarios y trabajadores de las IES son lectores, se asume que deberían serlo. En este ámbito, la lectura y escritura son reconocidas como medios para acceder al conocimiento, para lograr el aprendizaje y asimismo para sustentar el desarrollo de las competencias que garantizan la formación profesional. La lectura de textos académicos y materiales de trabajo son fundamentales para los estudiantes; los profesores sustentan su trabajo en la lectura y los trabajadores y funcionarios la realizan en el día a 
día. Este tipo de lectura, la que es cotidiana en la universidad, se hace para saber qué hacer, cómo hacerlo, por qué y para qué; conlleva la comprensión, propicia alguna reflexión y generalmente lleva a la recreación del conocimiento; sirve para aprender y para obtener información. Hay que considerar que en los medios universitarios también se lee para incrementar la cultura y construir la ciudadanía, aunque hay que decir que esto muchas veces se hace por obligación. Asimismo, los procesos de enseñanza-aprendizaje se fundamentan en diversas actividades que tienen a la lectura como el eje central. Para cerrar esta caracterización, es posible resumir que la búsqueda, obtención, recreación y difusión del conocimiento se logra sobre la base de procesos de lectura. La lectura así entendida es la que se conoce como lectura utilitaria.

Cuando se pregunta por la lectura se debe decir a qué tipo de lectura se refiere uno, porque además de la lectura utilitaria hay otro tipo de lectura, esa que se denomina lectura recreativa, lectura por gusto o lectura por placer (Kringelbach, Vuust y Geake, 2008). Regularmente, en este caso se habla de lectura de textos de literatura: cuentos, novelas, poemas, obras de teatro y tal vez ensayos. Dado que también se puede leer literatura científica por placer, por gusto o por recreación, se distingue esta lectura identificando por qué no se hace; así, es posible decir que cuando se lee por placer no se lee para cumplir una tarea, no por un requerimiento laboral, no para obtener información, no para aprender, no como una obligación, sino para un disfrute: por el simple goce. Realizar cotidianamente la lectura por placer se considera un buen hábito; de hecho, se habla del hábito de la lectura, refiriéndose a la lectura no utilitaria. Se han argumentado muchos beneficios de la lectura recreativa, ya que la mueve una motivación de alto nivel, con lo que se activa la imaginación y se generan procesos fisiológicos e intelectuales que naturalmente generan el desarrollo de competencias asociadas a las habilidades del pensamiento, fundamentales para el desarrollo de las competencias académicas (Cordón, 2016; Cremin, 2007; Parlette y Howard, 2010); es decir, que el hábito de la lectura - de esta que se debe hacer por placer- debería tener una práctica significativa entre los universitarios; es por esta razón que las IES se han venido incorporando gradualmente a la promoción de este tipo de lectura y cada vez se encuentran más programas y estrategias que tienen como fin el contagio, la motivación y de alguna manera coadyuvar al desarrollo del hábito de la lectura literaria, más allá de las obligaciones académicas y laborales (Beglar, Hunt y Kite, 2011; véase universidadeslectoras.org).

Las TIC han tenido presencia en la institución universitaria desde que aparecieron y su desarrollo conjunto está a la vista; sin embargo, ante la revolución tecnológica de las últimas décadas, a las TIC se les asocia con la calidad educativa, con la innovación en la educación y con la productividad científica, además de garantizar una capacidad profesional de los egresados acorde a los mercados ocupacionales. Nolasco-Vázquez y Ojeda-Ramírez (2016a) señalaron que el mejoramiento en estos aspectos en las IES depende, en principio, de las capacidades de la universidad para asimilar el uso de estos recursos; en su propuesta para medir la incorporación de las TIC a la universidad, concluyen que éstas son un instrumento que puede potenciar las capacidades de desarrollo mediante su uso efectivo. Observaron, a partir de una evaluación preliminar en un estudio de caso, Nolasco-Vázquez y Ojeda-Ramírez (2016b), que el mejoramiento de las capacidades de la institución universitaria asociadas con las TIC se fundamenta inicialmente en la visión y voluntad institucional para beneficiarse de la tecnología, y de si existen las condiciones para el uso efectivo en las funciones sustantivas y adjetivas; partiendo de esto, es posible decir que es un hecho incuestionable que las TIC están propiciando la reformulación de la de enseñanza-aprendizaje y que estos recursos permiten que la transmisión de información en los procesos formativos de la educación superior sea un asunto secundario. Los cursos ya, en muchas partes, se denominan "experiencias educativas", las modalidades y los enfoques para conducirlos se están diversificando, todo con el uso de los recursos que ofrecen las TIC. Por esto, es posible decir que el trabajo en las IES - tanto el académico como el de gestión- se desarrolla en gran medida con el uso intensivo de estas tecnologías. La imagen de un universitario, sea académico, trabajador o estudiante, es difícil de pensar sin una o varias pantallas, en las que fundamentalmente se lee y se escribe para realizar los más diversos procedimientos. Así, un universitario debe contar, por estos motivos, con una serie de habilidades y competencias informacionales para realizar con eficiencia su trabajo, o bien su proceso formativo si es 
un estudiante. López González y Ramírez Martinell (2016) construyeron algunas reflexiones acerca de las percepciones y valoraciones que tienen los profesores sobre la tecnología digital. Aplicaron una encuesta a 281 profesores de distintas facultades de la UV, preguntando sobre la conectividad, computadoras de escritorio, portátiles, dispositivos, periféricos, colecciones de texto completo especializadas en formato digital y otros servicios; asimismo, su relación con las políticas institucionales de utilización de las TIC. Identificaron las tendencias de equipamiento de los profesores universitarios y los orígenes de los recursos, lo que plantea una serie de retos para el equipamiento y la capacitación a fin de permitir una mejora de la apropiación y uso de los recursos tecnológicos. A este respecto, Cuevas Cerveró y Marzal García-Quismondo (2007) propusieron, desde la biblioteca, un modelo centrado en habilidades informativas para mejorar la competencia lectora en las IES, "como elemento holístico y vertebrador, convirtiendo de este modo a la lectura en instrumento indispensable para construir conocimiento". Argumentan que esto se justifica porque "se han producido cambios tan significativos como el aumento en la oferta lectora, la diversificación de los soportes de lectura y las nuevas formas de leer, escribir y comunicarse a través de Internet" (p. 49).

Los nuevos soportes -las pantallas- hacen que los textos ahora sean más accesibles, portables y tengan mayores niveles de complejidad, incorporando elementos como hipervínculos, imágenes y videos. A este respecto, Cordón-García y Jarvio Fernández (2015) presentan una revisión sobre la evolución de los soportes de lectura y escritura, analizando los cambios, así como el impacto en las relaciones comunicativas. Consideran que, debido al desarrollo tecnológico, la etapa actual es determinante para comprender las nuevas prácticas de lectura y escritura; estos autores concluyen que no se cuenta con suficientes evidencias que permitan sostener la posible desaparición del libro impreso, pero argumentan que sí se puede hablar de la aparición de nuevas formas de leer y escribir. Vaca Uribe (2009) reflexiona sobre las continuidades y rupturas de lectura en la era digital, en relación con las mismas prácticas en impreso. Señala que es necesario distinguir el medio de su uso, aseverando que lo importante es la vocación cultural con la que se usan, ya que: "Es el uso del medio y no el medio en sí mismo el que puede cambiar la era” (p. 31). Cordón (2016) argumenta que empieza a ocurrir con los libros lo que ya está pasando con las revistas, que es un proceso de migración de los soportes impresos a los digitales; es decir: "que el gradiente de las funcionalidades iba basculando progresivamente hacia lo digital en detrimento de lo físico, hasta que, llegado un punto, se abandonaba completamente el soporte de referencia” (p. 23). Gutiérrez (2009, p. 146) también planteó la necesidad de redefinir la lectura desde la perspectiva cultural, porque "ofrece la oportunidad de asumir que ésta no es una práctica, ni universal ni constante, y que realmente se produce históricamente”, sobre todo para tener evidencias de "las líneas y tensiones de fuerzas de la cultura que están y que, a futuro, estarán determinando la configuración de dicha práctica”.

Los estudios recientes sobre la comparación de la preferencia de los formatos de lectura son una evidencia del interés que se tiene en este tema. Moya y Gerber (2016) caracterizaron la lectura en formatos digitales en comparación con los impresos, analizando la lectura de libros, revistas, periódicos e historietas a partir de los datos de un estudio nacional en Chile; identificaron la afinidad existente en las prácticas lectoras en determinados grupos sociales así como un tipo de lector al que denominaron "lector omnívoro", al cual lo caracterizaron en términos de formatos digitales e impresos, de posiciones sociales aventajadas (alta educación e ingresos) y de padres con alta educación; concluyen que son ellos, los lectores omnívoros, quienes tienen una mayor disposición a leer distintos materiales y en distintos formatos. Taipale (2015), por su parte, usa la teoría de la práctica social para indagar sobre las diferencias de las prácticas de lectura y escritura comparando papel/lápiz con teclado/pantalla. Este investigador se enfocó en aspectos corporales de estas prácticas, considerándolas "técnicas del cuerpo", que a lo largo de los años se han visto más mediadas por las TIC. Analizó ensayos escritos de 25 estudiantes en la Universidad de Jyväskylä, Finlandia. Los resultados muestran que el papel se considera un material adecuado y adaptable a numerosos propósitos de lectura y posiciones corporales, mientras que con respecto a la pantalla, los lugares y puestos de lectura se consideran más limitados debido a las condiciones de los dispositivos electrónicos. Los estudiantes describen la escritura 
a mano como una práctica corporal más flexible y relajada que la mecanografía, pero se identifica que las habilidades de escritura están empeorando. Las habilidades y competencias resultaron ser menos decisivos que los materiales y las imágenes para explicar las diferencias en las prácticas de lectura y escritura. El estudio argumenta que las interfaces de escritura y de lectura digital no determinan de manera significativa las formas en que leemos y escribimos, como se ha venido sugiriendo. En esta misma línea, Tveit y Mangen (2014) realizaron una comparación de la lectura de una narración, considerando el texto literario en un dispositivo electrónico y la correspondiente versión impresa; se demostró que las preferencias están relacionadas con el sexo y con los hábitos generales de lectura. El estudio se desarrolló en un ámbito escolar y participaron 143 estudiantes de 15 años, 72 eran mujeres; se les pidió que comenzaran a leer una novela en un dispositivo y luego continuaran leyendo en el otro soporte. Se realizó una encuesta antes y después de la sesión de lectura, midiendo los hábitos de lectura en general, las preferencias del soporte y las experiencias con la lectura, tanto en la pantalla como en el papel. Los resultados mostraron que, en general, la mayoría de los estudiantes preferían leer en el soporte electrónico. Esta preferencia fue particularmente fuerte entre los varones y los lectores reacios, mientras que los lectores ávidos estuvieron más a favor de la versión impresa.

Muller, Hanson, Martínez y Meyer (2017) reportan una encuesta que se realizó en la Universidad Estatal de Sam Houston, entre los estudiantes, los académicos y los trabajadores para comprender mejor cómo la comunidad universitaria utiliza los materiales de la biblioteca para la lectura recreativa. Se encontró que se prefirieron los materiales impresos, con un interés creciente en otros formatos. Un número significativo de encuestados no vieron la biblioteca como una fuente de materiales recreativos de lectura; aquellos que sí, reportan que leyeron en una amplia gama de géneros. Concluyen que el estudio permitió una mejor comprensión de las preferencias de lectura recreativa, con lo que la biblioteca puede ampliar las estrategias que permitan satisfacer todas las demandas de los usuarios. Por su parte Seok y Da Costa (2017) exploraron las diferencias de género en la lectura de textos digitales e impresos. Investigaron los predictores de la preferencia digital junto con las diferencias de género considerando las percepciones hacia la lectura de textos impresos y digitales. Utilizando modelos de regresión identificaron relaciones significativas, donde el género fue un predictor de la preferencia digital; los hombres mostraron una mayor propensión hacia las TIC; la preferencia de los hombres hacia lo digital fue significativamente más alta que la de las mujeres. Al mismo tiempo, las percepciones de las mujeres indicaron que ellas leen en línea más con fines de entretenimiento y aprendizaje, y leen de forma más selectiva, mientras que los hombres leen materiales digitales más para fines de obtención de conocimiento.

Lent (2015), en una reseña del libro de ensayos editado por Socken (2013), hace un recorrido sobre las reflexiones de un grupo de académicos en torno a la pregunta de ¿por qué leer literatura en la era digital?, que se incluyen en este libro, titulado: The Edge of the Precipice. El recorrido va desde las posturas apocalípticas, como en los ensayos titulados Un mundo sin libros y El final de la lectura, donde se señala que la gente tiene a la lectura como una actividad marginal, ya que se prefiere ver la televisión, series, películas, y se dice que cuando se habla de leer, la gente se refiere a leer en internet, en los dispositivos, y en el mejor de los casos, dicen que periódicos o revistas. El recorrido va desde los que establecen que no deberían llevarse las cosas a ese extremo, argumentando que cada vez se lee más, y que las razones para leer literatura en la era digital prácticamente deberían ser las mismas por las que se leyó en otras épocas. Se hacen referencias a las tecnologías que han irrumpido en otras épocas: cuando surgió la imprenta, cuando apareció la radio y la televisión, y se argumenta sobre la postura de que hoy la lectura tiene más posibilidades, hablándose de la lectura digital con todas sus variantes. Por último, se plantea un futuro promisorio para el libro electrónico, pero no se deja de reconocer que, respecto a la lectura de literatura, "puede cambiar de formato, pero su función principal (...) que es compartir la imaginación, los sentimientos, la experiencia y los pensamientos de los demás, permanecerá”.

En resumen, es posible afirmar la pertinencia de los estudios sobre la preferencia de los soportes, y los factores asociados con las prácticas de lectura. Se acota esta visión retomando a Vaca Uribe (2009) que concluye su ensayo diciendo que: 
Los contenidos de los que serán portadores los diversos medios electrónicos en un futuro no muy lejano serán aquellos más "funcionales" o utilitarios (comercio, información en general, educación formal, comunicación personal) mientras que los textos más identificados con "la lectura sabia" o "la lectura hermenéutica, interpretativa" (incluida ahí la literatura) permanecerá primordialmente en papel: contenido tradicional, medio tradicional. Así, más que en una ruptura, debemos ubicar los textos electrónicos en un continuo histórico, al igual que las prácticas de lengua escrita, puesto que ahora hay que sumar a los usos textuales en papel, los usos "propiamente electrónicos" de los textos, muchos de los cuales desconocemos ahora. (p. 39).

Este trabajo forma parte de la línea de investigación "Representaciones sociales de la lectura digital en la Universidad Veracruzana”; tiene el propósito de explorar la preferencia por el texto impreso o digital para la lectura no utilitaria en una comunidad universitaria. Se sustenta en datos obtenidos en una encuesta realizada entre la comunidad de la Universidad Veracruzana (UV), institución que cuenta con el Programa Universitario de Promoción de la Lectura y diversos programas académicos que se orientan a esta temática, particularmente la Especialización en Promoción de la Lectura; asimismo, esta institución pública estatal forma parte de la Red Internacional de Universidades Lectoras (www.riul.com) y en ella se han realizado estudios previos (Castro, Jarvio Fernández, Garrido y Ojeda, 2008; Jarvio Fernández, 2011) que permiten valorar la evolución de esta práctica cultural en esta comunidad universitaria.

\section{Metodología}

La Universidad Veracruzana es una IES pública, distribuida en cinco regiones geográficas (Xalapa, Veracruz, Córdoba-Orizaba, Coatzacoalcos-Minatitlán y Poza Rica-Tuxpan) del Estado de Veracruz. Atiende a alrededor de 70 mil estudiantes (un poco más del 30\% de la matrícula de educación superior de este estado mexicano), con cerca de 7 mil académicos. Las regiones de Xalapa y Veracruz concentran aproximadamente el $50 \%$ y $25 \%$ del tamaño de la institución, con el $25 \%$ restante distribuido, más o menos, en la misma proporción en las otras regiones. Cuenta con más de 70 años de vida y en su historia reciente ha evolucionado a la modernidad incorporando intensivamente el uso de las TIC, lo que se puede constatar en sus documentos de planeación, desde donde se ha planteado la incorporación de estos recursos, considerando la dotación de infraestructura y equipamiento, conectividad y servicios tecnológicos para cada una de las entidades en las cinco regiones geográficas donde tiene presencia; se pueden identificar asimismo la implementación de programas de formación en TIC para el profesorado, la puesta en marcha de proyectos de innovación educativa, en los que se capacitó a los profesores para el diseño e impartición de experiencias educativas; cuenta en su haber el establecimiento de un área básica de formación que considera las TIC para todos los programas educativos que ofrece la institución; se ha promovido el uso de la biblioteca virtual, del sistema de información distribuida para la gestión institucional y de la plataforma EMINUS para la gestión de la enseñanza en línea y mixta, así como el uso de un sistema de videoconferencias, etc. (Morales Rodríguez, Ramírez Martinell y Excelente Toledo, 2015). Cuenta con un sistema bibliotecario con Unidades de servicios bibliotecarios y de información (USBI) en cada una de las principales ciudades del estado, las cuales funcionan como bibliotecas centrales.

En el año 2006, la UV lanzó una iniciativa sin precedentes en las universidades mexicanas: el Programa Universitario de Formación de Lectores (PUFL) (www.uv.mx/puf). El programa inició con la difusión de la Colección Biblioteca del Universitario (CBU) de textos literarios clásicos producidos por la Dirección Editorial de la UV y dirigida por Sergio Pitol (Premio Cervantes, 2005), y con la articulación de los esfuerzos que se venían desarrollando en la institución en pro de la promoción de la lectura. Durante los años 2007 al 2011 se dio gran impulso promoviendo actividades como el Festival de la Lectura, como también la articulación del PUFL con la Feria Internacional del Libro Universitario (FILU), un evento que tiene más de dos décadas de venirse realizando ininterrumpidamente. En el año 2007 el PUFL realizó una encuesta que dio cuenta de las prácticas lectoras y culturales en la comunidad de la UV (Castro, Jarvio, Garrido y Ojeda, 
2008), lo que permitió identificar áreas de oportunidad para el desarrollo del programa. Pronto empezaron a aparecer iniciativas, como los talleres de promoción de la lectura, la creación de Martes de Lectores y Lecturas y la actividad semanal Música y Literatura, las experiencias educativas (EE) Taller de Promoción de la Lectura (Pérez-Ornelas, 2015) y Música y Literatura -en el marco del Área de Formación de Elección Libre (AFEL) del Modelo Educativo Integral y Flexible (MEIF) - que pueden ser cursadas por los estudiantes de cualquier licenciatura de la UV. Durante 2010 se realizó la encuesta sobre la lectura digital en la UV, a partir de cuyos resultados se concluyó que:

Del uso masivo de las nuevas tecnologías, son pocas las aplicaciones que se realizan con estos recursos en el ámbito de la lectura. Sin embargo existe una tendencia en el incremento tanto de estas aplicaciones, como en la integración hacia nuevas formas de comunicación incluyendo a la lectura digital con todas sus implicaciones (Jarvio Fernández, 2011, párrafo final del resumen).

Durante los meses de abril y mayo de 2017, se aplicó un cuestionario en línea para obtener información sobre las prácticas lectoras de los universitarios de la UV; el instrumento fue contestado por un poco más de 1400 miembros de la comunidad que fueron clasificados por área académica, región universitaria, ocupación y sexo.

\begin{tabular}{|c|c|c|}
\hline Nombre de la variable & Descripción & Categorías de respuesta \\
\hline SEX & Sexo del encuestado. & Femenino $(F)$ y Masculino $(M)$ \\
\hline OCUP & $\begin{array}{l}\text { De acuerdo a las funciones que } \\
\text { desempeña dentro de la } \\
\text { institución, será la ocupación. }\end{array}$ & $\begin{array}{l}\text { Académico (académicos, } \\
\text { académicos ejecutantes, docentes } \\
\text { de tiempo completo y por } \\
\text { asignatura, investigadores, } \\
\text { técnicos), Empleado } \\
\text { (administrativo y de biblioteca), } \\
\text { Estudiante (de licenciatura, de } \\
\text { posgrado y egresados), y } \\
\text { Funcionario (de administración } \\
\text { universitaria y de entidad } \\
\text { académica). }\end{array}$ \\
\hline REG & $\begin{array}{l}\text { Región universitaria de } \\
\text { adscripción del encuestado. }\end{array}$ & $\begin{array}{l}\text { Xalapa y Otro (Incluye Veracruz- } \\
\text { Boca del Rio, Poza Rica-Tuxpan, } \\
\text { Córdoba-Orizaba, Coatzacoalcos- } \\
\text { Minatitlán-Acayucan). }\end{array}$ \\
\hline AREA & $\begin{array}{l}\text { Área de adscripción del } \\
\text { encuestado. }\end{array}$ & $\begin{array}{l}\text { Artes y Humanidades (AH), } \\
\text { Biológico-Agropecuaria (BA), } \\
\text { Ciencias de la Salud (CS), } \\
\text { Económico-Administrativa (EA) y } \\
\text { Técnica (T). }\end{array}$ \\
\hline GUSTO & $\begin{array}{l}\text { El nivel de gusto declarado por el } \\
\text { encuestado hacía la lectura. }\end{array}$ & $\begin{array}{l}\text { Nada y poco (1), Regular (2) y } \\
\text { Mucho (3). }\end{array}$ \\
\hline HLL & $\begin{array}{l}\text { Horas que durante la última } \\
\text { semana el encuestado declaró } \\
\text { haber dedicado a la lectura de } \\
\text { literatura, indistintamente del } \\
\text { formato. }\end{array}$ & $\begin{array}{l}\text { No leí (0), hasta } 2 \text { horas }(1) \text {, de } 3 \\
\text { a } 5 \text { horas (2) y más de } 5 \text { horas } \\
\text { (3). }\end{array}$ \\
\hline PREF & $\begin{array}{l}\text { Definen el formato en que se } \\
\text { prefiere leer libros de literatura; } \\
\text { entre ellos el cuento, novela y } \\
\text { poesía, etc. }\end{array}$ & Digital, Impreso y Cualquiera. \\
\hline SIG & $\begin{array}{l}\text { Explican lo que es real mente la } \\
\text { lectura para cada uno de ellos. }\end{array}$ & $\begin{array}{l}\text { Es una pregunta abierta, con } \\
\text { respuesta en texto. }\end{array}$ \\
\hline CAL & $\begin{array}{l}\text { De acuerdo a la respuesta que el } \\
\text { encuestado ha brindado en el } \\
\text { significado de la lectura, se ha } \\
\text { hecho una clasificación. }\end{array}$ & $\begin{array}{l}\text { Concepto básico o no declaró (1), } \\
\text { concepto convencional (2) y } \\
\text { concepto excelso (3). }\end{array}$ \\
\hline
\end{tabular}

TABLA 1

Descripción de las variables utilizadas en los análisis de este estudio

En la Tabla 1 se presentan las variables que se consideraron para este estudio. Después de un proceso de limpieza, eliminando casos incompletos, la base de datos que se sometió a análisis estadísticos contó con 1311 registros. Asimismo, se pueden ver los nombres y las categorías de los indicadores utilizados en los análisis: GUSTO, HLL, PREF y CAL. Para la realización de los análisis se obtuvieron las distribuciones de 
frecuencias de las variables una a una y se estudió la significancia de las asociaciones de parejas de variables, considerando a la preferencia del tipo de soporte para leer literatura como variable respuesta, lo que se realizó a partir de la prueba Ji-Cuadrada. Una vez identificadas las asociaciones significativas se realizó un análisis de correspondencia múltiple (Nenadic' y Greenacre, 2007) para explorar las asociaciones multivariantes.

Las figuras 1 y 2 presentan la distribución de la muestra de acuerdo a las variables de clasificación.

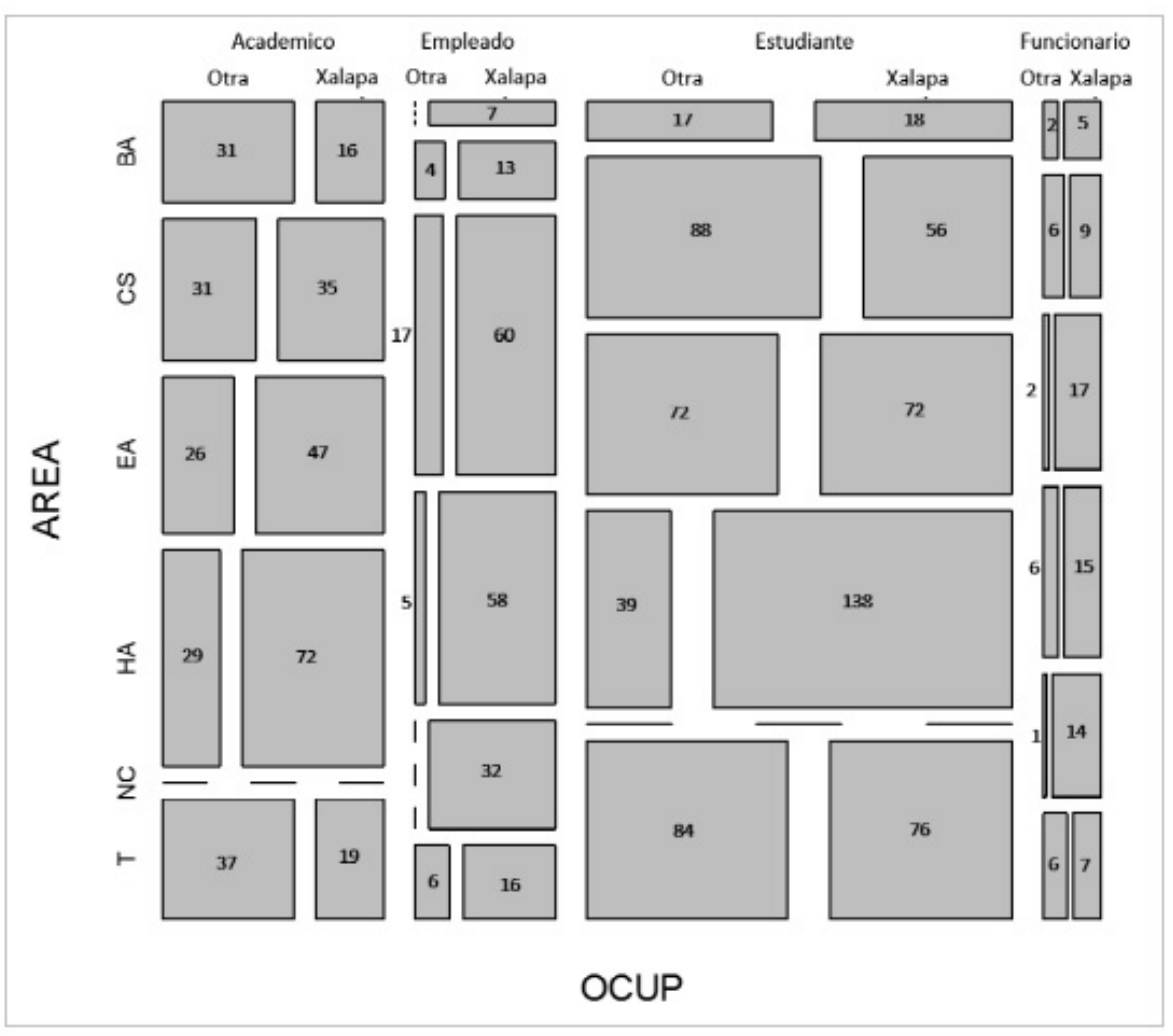

FIGURA 1

Distribución de los 1311 encuestados según la clasificación por región, ocupación y área académica. Los casos NC corresponden con empleados y funcionarios que no tienen adscripción a área académica 


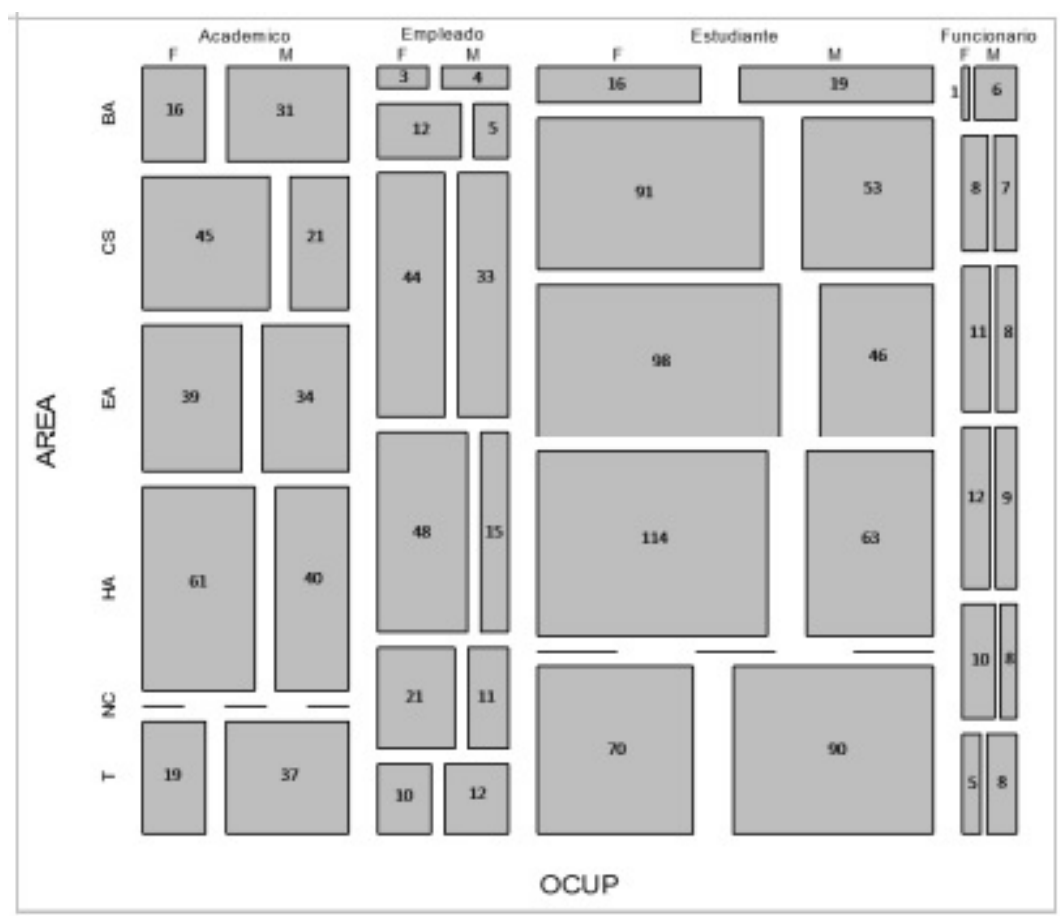

FIGURA 2

Distribución de los 1311 encuestados según la clasificación por sexo, ocupación y área académica. Los casos NC corresponden con empleados y funcionarios que no tienen adscripción a área académica

\section{Resultados y Discusión}

El principal resultado de este estudio es que 7 de cada 10 de los encuestados prefirió el texto impreso para la lectura no utilitaria; entre los de sexo masculino la proporción decrece hasta 6 de cada 10, lo que constituye evidencia estadística altamente significativa de la asociación del sexo con la preferencia (ver Figura 3). 


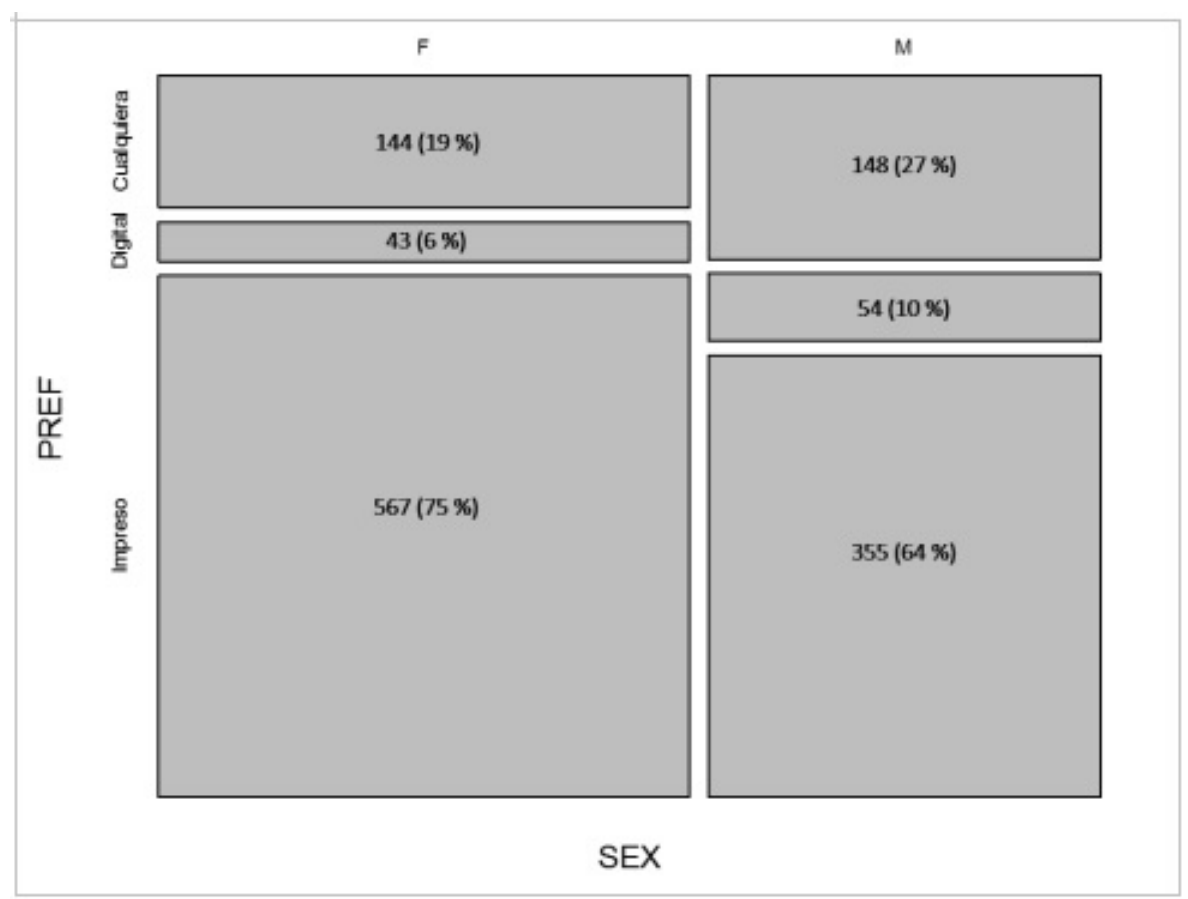

FIGURA 3

Distribución de la preferencia del formato por sexo (Ji-Cuadrada=20.92, $\mathrm{gl}=2, \mathrm{p}<0.001$ )

Cuando la preferencia se cruzó con área académica (Ji-Cuadrada $=11.409, \mathrm{gl}=10, \mathrm{p}=0.327$ ) y ocupación (Ji-Cuadrada $=8.7751, \mathrm{gl}=6, \mathrm{p}=0.187$ ) no se observaron asociaciones significativas; el cruce con región (JiCuadarada $=5.0112, \mathrm{gl}=2, \mathrm{p}=0.082$ ), resultó con una significancia leve, con una proporción de $73 \%$ que prefiere el texto impreso en la región de Xalapa, lo cual no resulta realmente significativo.

El indicador de tiempo dedicado a la lectura por ocupación presentó diferencias estadísticamente significativas, mostrándose que hay un patrón que muestra mayor tiempo de dedicación a la lectura no utilitaria entre los académicos y los estudiantes, seguidos de los funcionarios, como se puede observar en la Figura 4. 


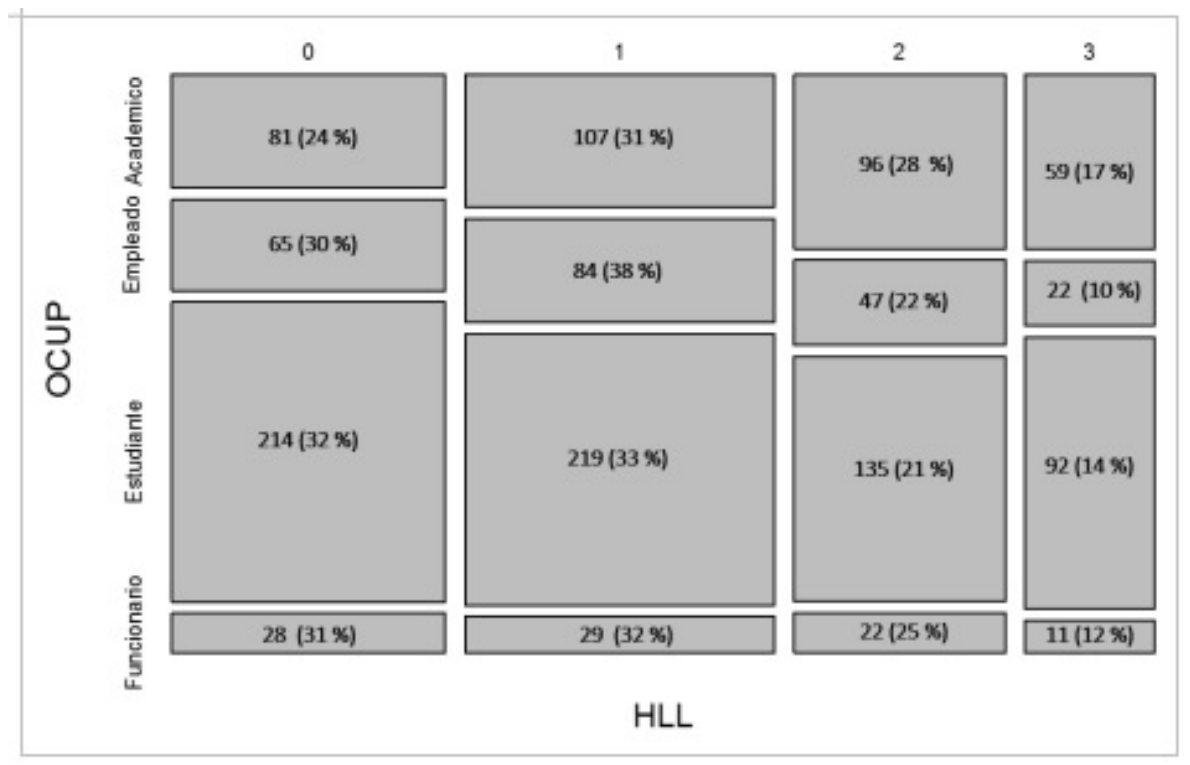

FIGURA 4

Distribución de la ocupación por las horas dedicadas a la lectura de literatura (Ji-Cuadrada $=19.21, \mathrm{gl}=9, \mathrm{p}=0.023$ )

Respecto al gusto por la lectura se obtuvo que los académicos y los funcionarios declararon, en una proporción de 7 y de 6 de cada 10, respectivamente, que les gusta mucho la lectura de literatura, contra 5 y 4 de cada 10 entre los empleados y los estudiantes, respectivamente. Esta asociación es estadísticamente significativa, como se puede constatar en la Figura 5.

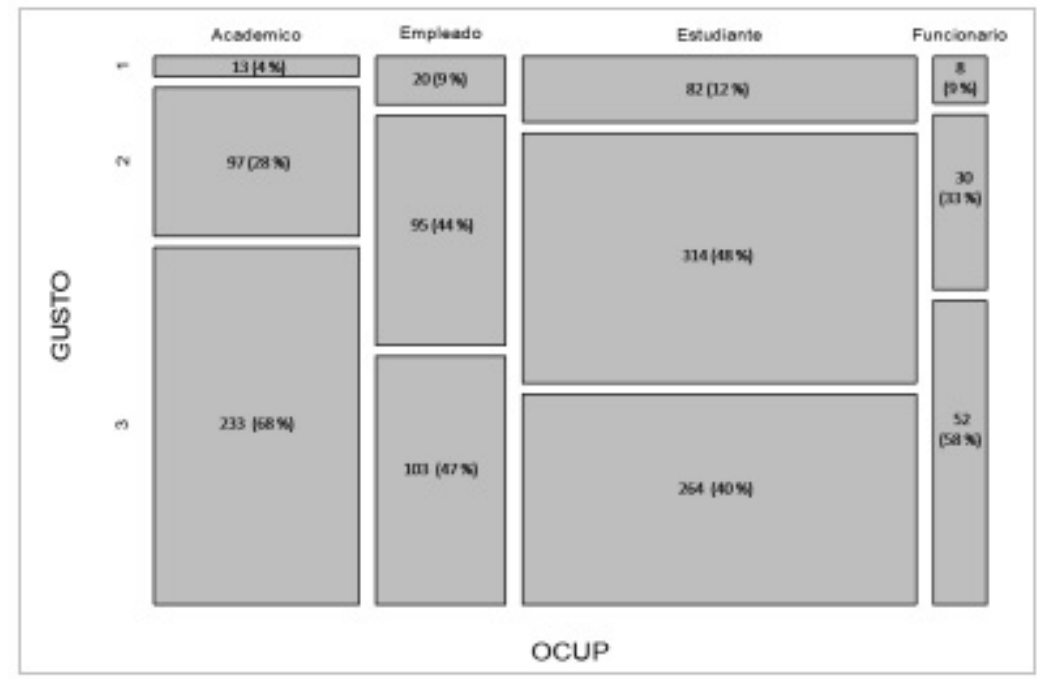

FIGURA 5

Distribución del gusto hacía la lectura por la ocupación (Ji-Cuadrada $=77.02, \mathrm{gl}=6, \mathrm{p}<0.001$ )

En lo que se refiere a los conceptos de lectura, los más básicos o de ausencia de opinión se presentaron entre los estudiantes y los empleados, mientras que los más elaborados aparecieron en el grupo de los académicos y los funcionarios; esta relación resultó estadísticamente significativa como puede verse en la Figura 6. 


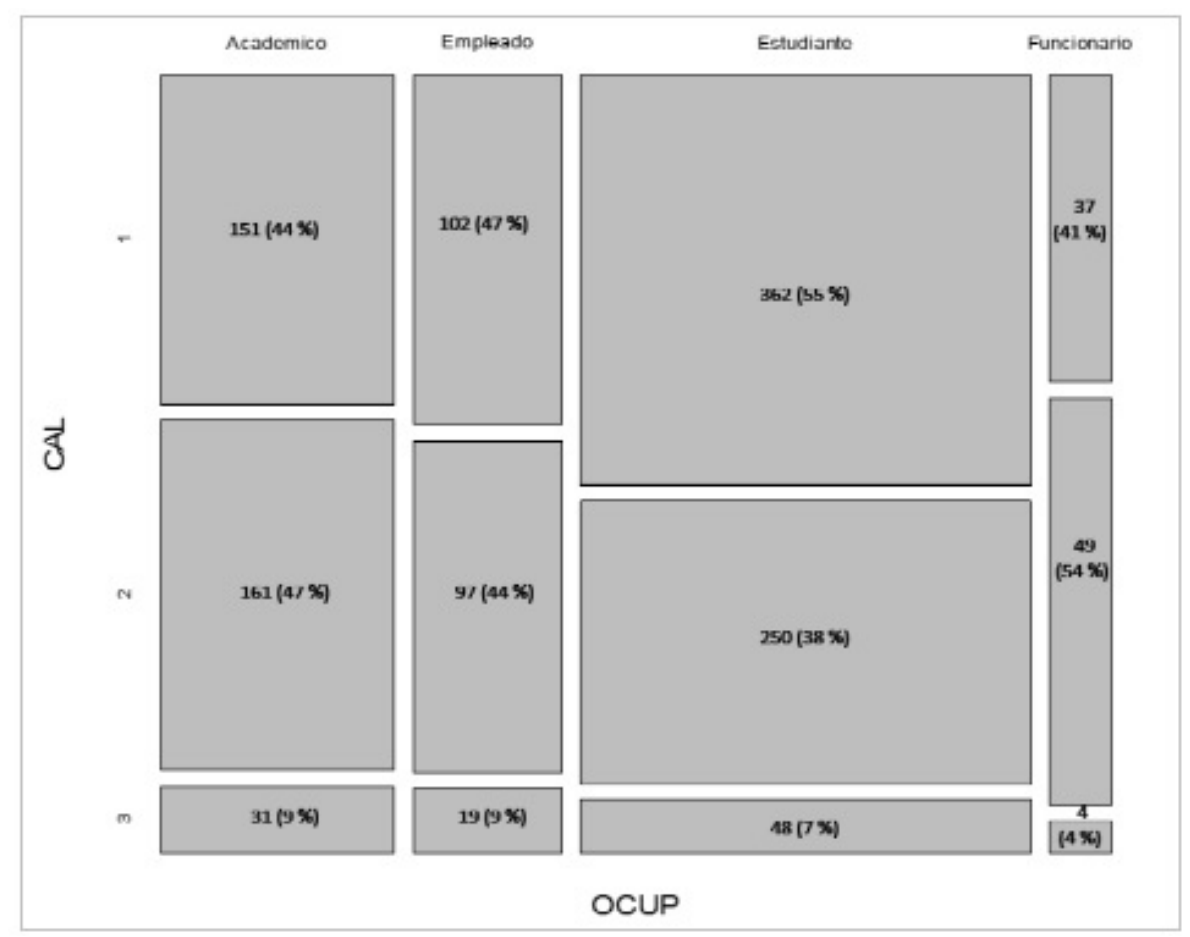

\section{FIGURA 6}

Distribución de la calificación sobre el concepto de lectura por la ocupación (Ji-Cuadrada $=18.03, \mathrm{gl}=6, \mathrm{p}=0.006$ )

Como era de esperarse, al relacionar el gusto por la lectura con las horas dedicadas a esta actividad en la última semana se presentó una asociación altamente significativa, como puede constatarse en la Figura 7.

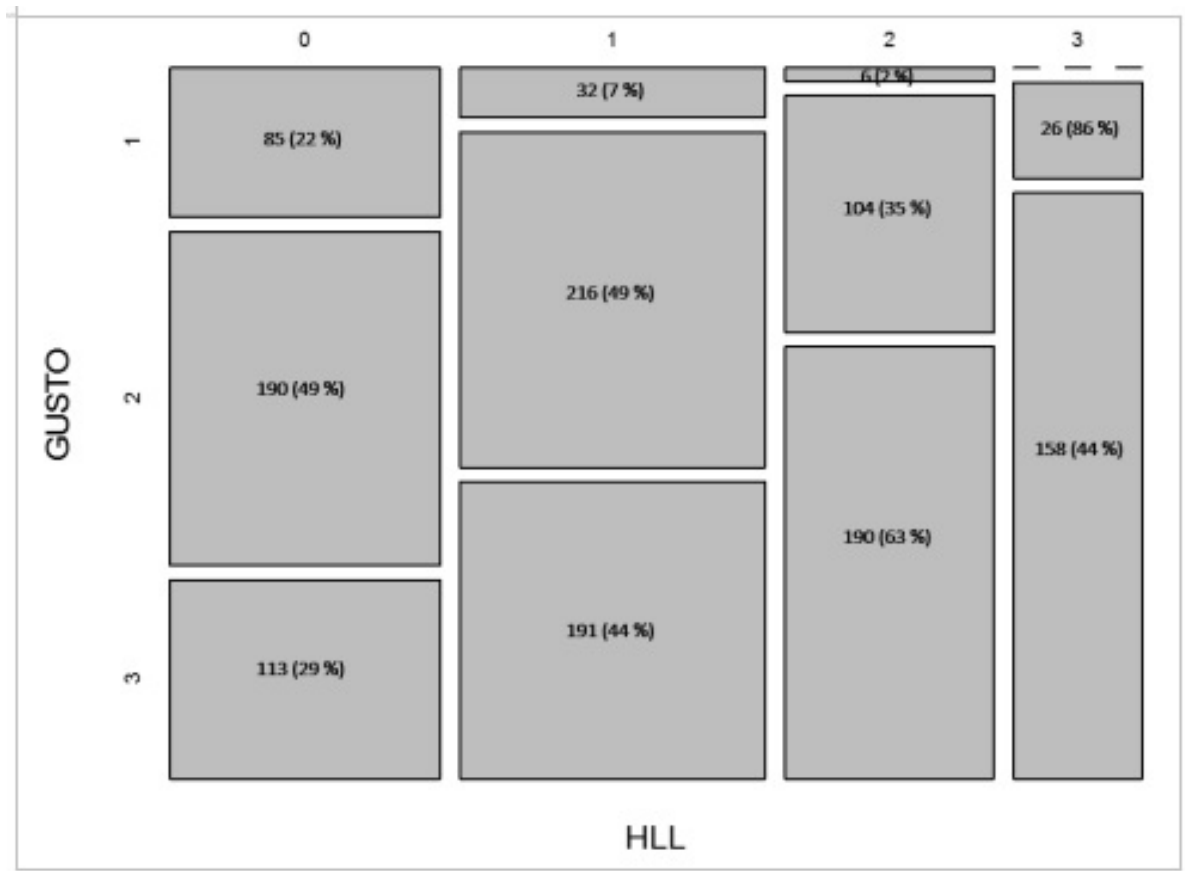

FIGURA 7

Distribución del gusto hacía la lectura por las horas que declararon haber dedicado en la última semana (Ji-Cuadrada=246.33, $\mathrm{gl}=6, \mathrm{p}<0.001$ ) 
$\mathrm{Al}$ realizar el análisis multivariante se encontraron algunas asociaciones a destacar: los que dijeron dedicar menos tiempo a la lectura tienden a preferir cualquier formato y tienden a declarar que les gusta poco o nada la lectura de literatura, mientras que los que dijeron que le dedicaron más tiempo a la lectura de literatura son mayoritariamente los que dijeron que les gusta mucho leer, con mayor frecuencia de académicos y funcionarios. Esto puede verse en la Figura 8, que tiene una representación de un poco más del $70 \%$ de la variabilidad de los datos.

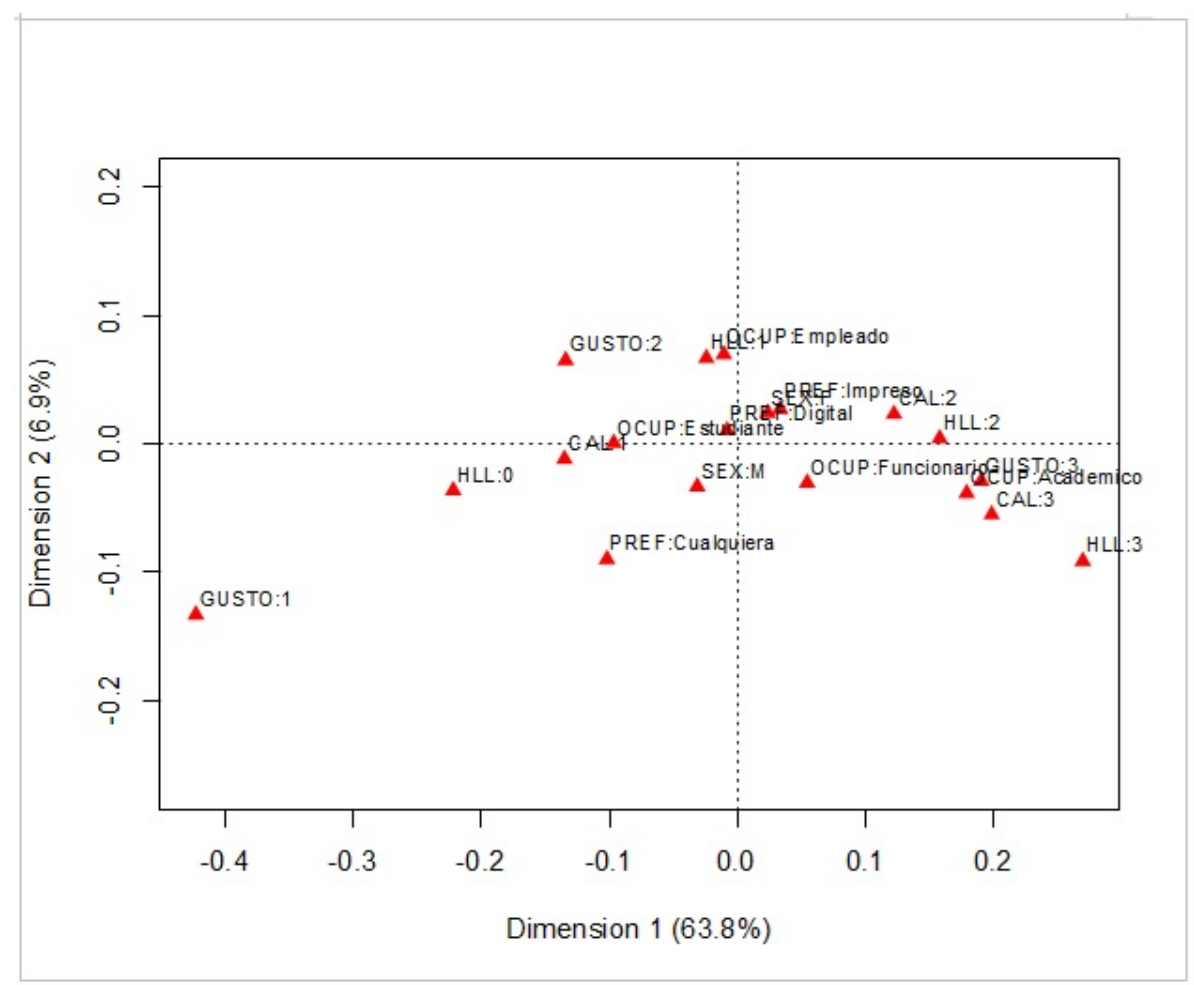

FIGURA 8

Mapa de correspondencia entre las categorías de las variables sexo, ocupación, gusto, horas dedicadas a la lectura de literatura y preferencia del formato

$\mathrm{Al}$ analizar los conceptos de lectura vertidos por aquellos que declararon que prefieren el formato impreso, como puede verse en la Figura 9, se resalta "aprender" y "conocimiento", que son palabras más asociadas a la lectura utilitaria. Sin embargo, "entretenimiento", "imaginar", "viajar", "cultura", "abrir mundos", etc., también aparecen, aunque claramente en segundo término. Esto genera una serie de hipótesis que implican realizar una minería de datos más profunda. 


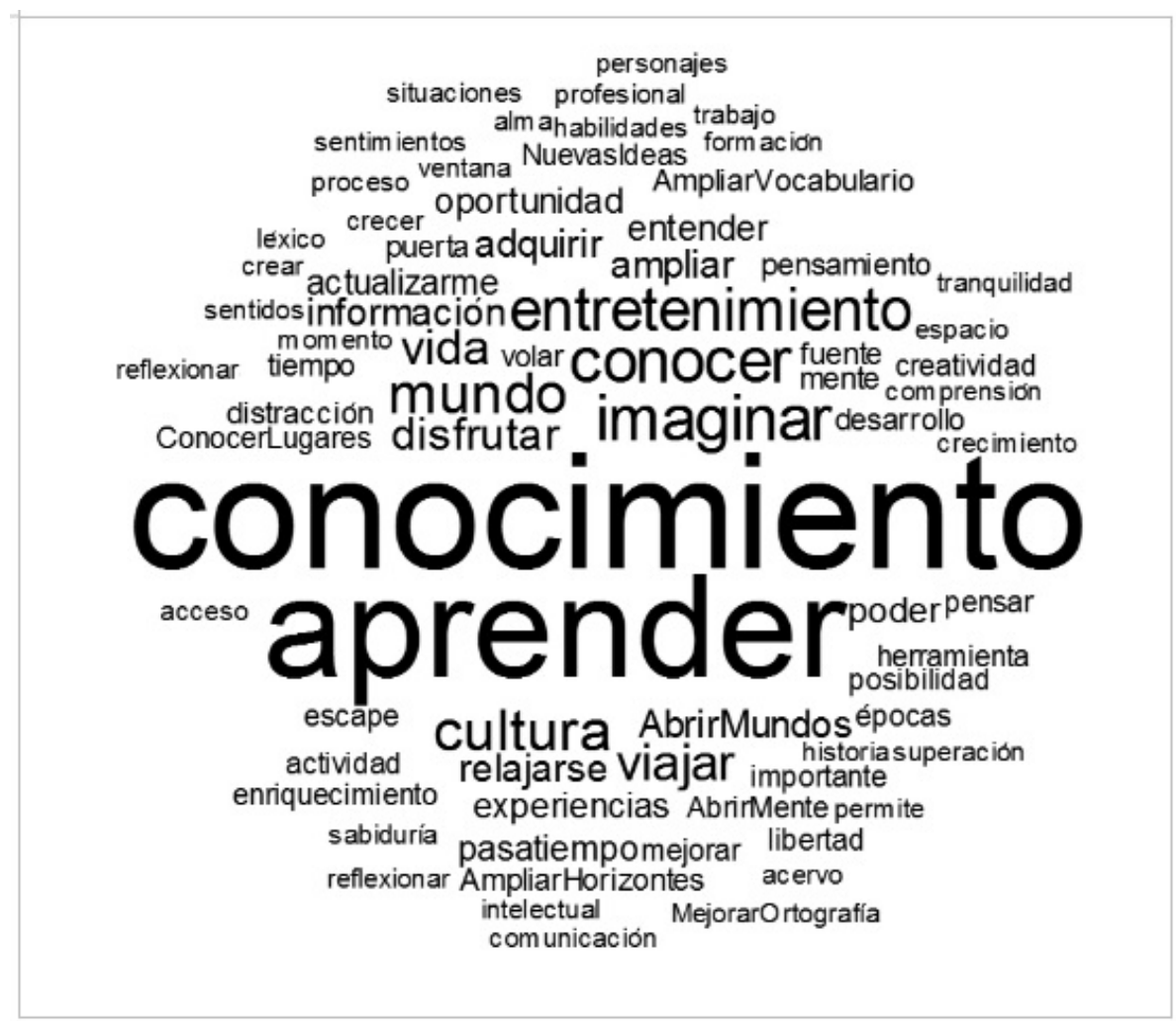

FIGURA 9

Nube de palabras acerca de lo que significa la lectura para los encuestados que eligieron como preferencia el formato impreso

\section{Conclusiones}

Hay que partir del hecho de que la comunidad estudiada está sometida a un conjunto de actividades permanentes de promoción de la lectura no utilitaria, ya que el PUFL (www.uv.mx/lectores lecturas) realiza talleres, presentaciones, coloquios y se imparten experiencias educativas para formar promotores de lectura no utilitaria; asimismo se realizan múltiples intervenciones en diferentes ámbitos y entidades, desde el programa de posgrado de especialización (www.uv.mx/epl); de la misma manera, hay que resaltar que la UV realiza cada año una feria del libro universitario, que promueve prioritariamente la literatura, además de que la editorial institucional tiene una añeja tradición promoviendo autores de literatura. Por esta razón, al estudiar la lectura no utilitaria se espera altos índices de gusto ( 5 de cada 10 respondieron que les gusta mucho) y dedicación (casi 3 de cada 10 dedicaron en la última semana más de 5 horas a la lectura de literatura). Cuando leemos los resultados a la luz de los antecedentes se percibe una evolución. Según Castro et al. (2008), 8 de cada 10 universitarios leían dando como razón principal aprender e informarse (p. 133 y p. 135), y sólo 2 de cada 10 leían por la recreación o el gusto (p. 69). En aquella ocasión no se preguntó explícitamente por la lectura no utilitaria, o por la lectura de literatura. En el contexto institucional para aquellas fechas la lectura digital era predominantemente utilitaria, ya que 7 de cada 10 usaban las TIC para leer en relación con las actividades académicas, para informarse, para estudiar, etc. (p. 113). El uso de las tabletas digitales era incipiente, y los teléfonos móviles no tenían todas las funcionalidades de los actuales, así que la lectura de literatura se hacía fundamentalmente en formato impreso. Al estudiar concretamente la lectura digital, Jarvio Fernández (2011) reportó que: "respecto a la preferencia de formato para leer libro de literatura, el impreso obtiene el mayor número de respuestas (86\%) y por el digital solo responde un 9\% de los encuestados" (p. 
114). Como puede verse, las cosas han cambiado ligeramente, pero el predominio del formato impreso sigue siendo claro, lo cual indica que dentro de la UV es importante implicarse de manera decidida en estrategias que permitan la formación en competencias que garanticen un mayor uso de la tecnología, incluso para la lectura de literatura.

Los conceptos sobre la lectura y las razones por las que se lee parecen seguir siendo las mismas; los análisis realizados no permiten una descripción de los detalles sobre en qué aspectos concretos se está cambiando, por lo que se deben realizar análisis más exhaustivos de los datos para hurgar en preguntas como qué se lee, cómo se lee, cuánto se lee y la relación de estas variables con el concepto que se tiene de lectura. Parece ser que la hipótesis de que la lectura de literatura, conceptualizada esta como recreativa, placentera y por gusto, se sigue conceptualizando de la misma manera, pero asimismo cómo dice Lent (2015, p. 2), citando uno de los ensayos reseñados, "la literatura, puede cambiar de formato, pero su función principal (...) que es compartir la imaginación, los sentimientos, la experiencia y los pensamientos de los demás, permanecerá”.

\section{REFERENCIAS}

Beglar, D., Hunt, A., y Kite, Y. (2011). The effect of pleasure reading on japanese university EFL learners' readings rates. Language Learning, 1-39. https://doi.org/10.1111/j.1467-9922.2011.00651.x

Castro, C., Jarvio, O., Garrido, F., y Ojeda, M. (2008). Prácticas lectoras en la Universidad Veracruzana: una encuesta. Xalapa, Veracruz: Universidad Veracruzana.

Cordón, J. A. (2016). La lectura en el entorno digital: nuevas materialidades y prácticas discursivas. Revista Chilena de Literatura, 94, 15-38.

Cremin, T. (2007). Revisiting Reading for pleasure: diversity, delight and desire. In Goouch, K., y Lambirth, A. (Eds.), Understanding Phonics and the teaching of reading (pp. 166-190). Berkshire: McGraw Hill.

Cordón-García, J. A., y Jarvio Fernández, A. O. (2015). ¿Se está transformando la lectura y la escritura en la era digital? Revista Interamericana de Bibliotecología, 38(2), 137-145. https://doi.org/10.17533/udea.rib.v38n2a05

Cuevas Cerveró, A., y Marzal García-Quismondo, M. A. (2007). La competencia lectora como modelo de alfabetización en información. Anales de Documentación, 10, 49-70.

Gutiérrez, E. (2009). Leer digital. La lectura en el entorno de las nuevas tecnologías de la información y la comunicación. Signo y Pensamiento, 54, 144-163.

Jarvio Fernández, A. O. (2011). La lectura digital en el ámbito de la Universidad Veracruzana (Tesis de Doctorado). Universidad de Salamanca. Departamento de Biblioteconomía y Documentación, Salamanca, España.

Kringelbach, M. L., Vuust, P., y Geake, J. (2008). The pleasure of reading. Interdisciplinary Science Reviews, 33(4), 321-335. https://doi.org/10.1179/174327908X392889

Lent, A. (2015). [Revisión del libro The Edge of the Precipice-Why Read Literature in the Digital Age?, porPaul Socken (ed.)]. Publishing Research Quarterly, 31(4), 330-331. https://doi.org/10.1007/s12109-015-9433-4

López González, R., y Ramírez Martinell, A. (2016). Políticas de equipamiento tecnológico en Educación Superior: reflexiones y orientaciones. Debate Universitario, 9, 53-67.

Morales Rodríguez, A. T., Ramírez Martinell, A., y Excelente Toledo, C. B. (2015). Apropiación de las TIC en la educación superior: una mirada desde la disciplina del profesorado. Research in Computing Science, 108, 45-53.

Moya, C., y Gerber, M. M. (2016). La lectura en formatos digitales en el Chile actual: nuevas prácticas y viejas desigualdades. Revista Chilena de Literatura, 94, 59-77.

Muller, K. L., Hanson, M., Martínez, M., y Meyer, L. (2017). Patron preferences: Recreational reading in an academic library. The Journal of Academic Librarianship, 43, 72-81.

Nenadic', O. y Greenacre, M. (2007). Correspondence analysis in R, with two- and tree-dimensional graphics: The ca package. Journal of Statistical Software, 20(3), 1-13. 
Nolasco-Vázquez, P., y Ojeda-Ramírez, M. (2016a). La evaluación de la integración de las TIC en la educación superior: fundamento para una metodología. RED. Revista de Educación a Distancia, 48(9). Recuperado de ht tp://www.um.es/ead/red/48

Nolasco-Vázquez, P., y Ojeda-Ramírez, M. M. (2016b). Una aproximación a un sistema de indicadores para evaluar la integración de las TIC en las instituciones de educación superior. Areté. Revista Digital del Doctorado en Educación de la Universidad Central de Venezuela, 2(4), 63-89.

Parlette, M., y Howard, V. (2010). Personal growth, habits and understanding: Pleasure reading among first-year university students. Evidence Based Library and Information Practice, 5(4), 53-67.

Pérez-Ornelas, T. (2015). Experiencia educativa Taller para promotores de lectura en la UV: alcances y logros (Trabajo recepcional de especialización). Universidad Veracruzana, México.

Seok, S., y Da Costa, B. (2017). Gender differences in teens' digital propensity and perceptions and preferences with regard to digital and printed text. TechTrends, 61, 171-178. https://doi.org/10.1007/s11528-016-0134-4

Socken, P. (ed) (2013). The Edge of the Precipice-Why Read Literature in the Digital Age? Montreal and Kingston, Canada: McGill-Queen's University Press.

Taipale, S. (2015). Bodily dimensions of reading and writing practices on paper and digitally. Telematics and Informatics, 32, 766-775.

Tveit, A. K., y Mangen, A. (2014). A joker in the class: Teenage readers' attitudes and preferences to reading on different devices. Library \& Information Science Research 36, 179-184.

Vaca Uribe, J. (2009). ¿Son nuevos los medios y los lectores en la era digital? Lectura y Vida, 30(2). Recuperado de ht tp://www.lecturayvida.fahce.unlp.edu.ar/numeros/a30n2/30_02_Vaca.pdf 